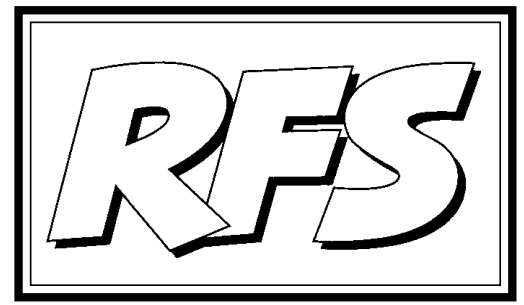

Revista de Fomento Social, 53 (1998), 31-46

\title{
Globalización y localismo: nuevas oportunidades para el desarrollo
}

El artículo trata de poner de manifiesto la relevancia del ámbito local en el marco del proceso de globalización. Esto se pone en evidencia tanto en la esfera económica, como en la social y la política. Más concretamente, se argumenta que el municipio ofrece una nueva estructura de oportunidades para la puesta en marcha de procesos de desarrollo económico, político y social. A esta tendencia, que junto a la globalización, articula las dinámicas y procesos de cambio en las sociedades contemporáneas, se le denomina nuevo localismo.

Clemente J. NAVARRO YÁÑEZ (*)

(*) Investigador del Instituto de Estudios Sociales Avanzados de Andalucía, centro del Consejo Superior de Investigaciones Científicas en Córdoba. Profesor asociado de la Universidad Pablo de Olavide (Sevilla). 


\section{Introducción}

Al mismo tiempo que la ciencia social atiende a procesos de carácter global, se descubre la especificidad y potencialidad del ámbito local en los procesos de reestructuración política, económica y social que tienen lugar en las sociedades contemporáneas. La práctica y puesta en marca de procesos de desarrollo local son cada vez más extensas y variadas. Las identidades ciudadanas tienden a localizarse, poniendo incluso en peligro las de carácter más amplio, tal y como la nacional. Y los gobiernos municipales parecen gestionar cada vez más parte de la política social.

Lo anterior, al menos, debe llamar la atención de los científicos sociales para que traten al ámbito local, junto al estatal y mundial, como unidad adecuada de análisis. Pero no sólo por lo que las dinámicas localistas, y por tanto particularistas, puedan afectar a otras de carácter más universalista, sino también porque el propio proceso de mundialización (1) parece estar dando lugar al desarrollo de una nueva estructura de oportunidades para la redefinición del papel de los gobiernos y sociedades municipales de cara a la puesta en marcha de procesos de desarrollo económico, político y social. A este conjunto de oportunidades es a lo que aquí se denominará nuevo localismo.

Desde nuestro punto de vista, este hecho puede constatarse apoyándonos en tres líneas argumentales. Por un lado, el hecho de que los procesos económicos, políticos y sociales producen impactos diferentes según la escala donde incidan, con independencia de otros factores o variables. Por otro, el hecho de que la comunidad local, y en concreto la ciudad, sigue constituyendo la unidad básica de convivencia. Por último, las potencialidades que ofrece la escala local para el desarrollo de procesos de participación ciudadana y democratización. Evidentemente, estos tres aspectos se encuentran estrechamente relacionadas entres sí, y en adelante, se tratarán de forma separada exclusivamente con fines de carácter analítico.

\section{Las bases sociales del desarrollo}

La constatación de las tendencias hacia la mundialización, y en concreto las relacionadas con los procesos de desarrollo y localización de las actividades

(1) En adelante se tomarán como sinónimos mundialización y globalización. 
económicas, ha hecho que se diagnostique la reducción, o incluso la incapacidad de los actores locales para afrontar los problemas derivados de los procesos de globalización. Desde este punto de vista los procesos locales son determinados por las tendencias de carácter general, sin que los actores puedan incidir en ellos, al menos de una forma significativa.

Tanto para la teoría marxista clásica, en su versión de la división internacional del trabajo y la teoría de la dependencia (Frank, 1972; Amin, 1972), como para la neo-marxista, y su teorización acerca de los procesos urbanos de consumo colectivo, él ámbito local no muestra ningún tipo de especificidad con respecto a otras escalas. Los gobiernos municipales, al igual que los centrales, de los que no son más que una parte, cumplen una función de protección y legitimación del sistema de acumulación capitalista. En todo caso, se distancia de otros ámbitos por el hecho de constituir el campo apropiado para la reproducción de la fuerza de trabajo mediante prácticas de consumo colectivo, concretamente, a través de la provisión de servicios: vivienda, educación y servicios sociales fundamentalmente (2).

El declive de la producción industrial y de las áreas urbanas industrializadas a partir de los años 70, así como el crecimiento de sectores productivos ligados al desarrollo tecnológico y la búsqueda de nuevas estrategias de localización económica, han contribuido a anunciar la crisis del «régimen de acumulación fordista» y la aparición de un «régimen de acumulación flexible» (Aglietta, 1974). Parte de la literatura que aborda este problema incide, al igual que las interpretaciones marxistas anteriores o la teoría clásica de lugar central, en la incapacidad de acción autónoma por parte de los actores locales. La gran movilidad del capital financiero, sector punta en el proceso global de reestructuración económica, hace que las localidades deban establecer relaciones de competencia para atraer sectores productivos, sin que exista posibilidad de desarrollo económico o social de carácter autónomo o endógeno (3).

Frente a estos diagnósticos de carácter estructuralista y de marcado determinismo economicista (4), otros puntos de vista señalan las potencialidades del

(2) Este punto de vista fue desarrollado originariamente por CASTELLS (1976), y ampliado posteriormente por CockBuRn (1977) y DunLEAvy (1980).

(3) Para una exposición más detallada de estos argumentos se remite a PIORE y SABEL (1984) y SASSEN (1990).

(4) El primer aspecto ha sido puesto de manifiesto, por ejemplo, por HAMPTON (1991: 235), el segundo por Stoker (1990) y Pretecille (1994). 
ámbito local. En primer lugar, porque los procesos de reestructuración económica no son simplemente «adaptación flexible» a problemas globales. Los actores locales los especifican, reaccionan de forma diferente e incluso pueden influir en ellos. Más aún, los patrones institucionales, normas y valores sociales propios de cada localidad actúan como filtro de los procesos que tiene lugar fuera su propio ámbito, y han mostrado su capacidad para establecer pautas de desarrollo económico y social de carácter relativamente autónomo, como en el caso de la «Terza Italia» (Trigilia, 1981; Bagnasco, 1977) o más recientemente en América Latina (Pérez Sáinz, 1977; Lambi y otros, 1997).

Esto evidencia que los procesos de restructuración no tienen un carácter estrictamente económico y dependen en buena parte del conocimiento y capacidades de los habitantes locales, así como de su capital cultural y social (5). A ello se une que las respuestas a la globalización se articulan a través de decisiones de índole político, en las que se definen las estrategias a seguir. Estas dan lugar a procesos diferenciales de localización económica, y por tanto, al establecimiento de distintos tipos de redes y sistemas de ciudades (Lipietz, 1993; Lafontaine, 1991).

Asimismo, la discusión acerca de los procesos de reestructuración urbana afectan directamente, tanto en términos cualitativos como cuantitativos, a la provisión de servicios por parte de los gobiernos locales. Ello ha provocado un cambio en las estrategias políticas en relación a la localización de las competencias en la provisión de éstos. Estas se concretan actualmente en la progresiva tendencia a la descentralización desde el estado central a los gobiernos locales.

\section{La relevancia de la vecindad e identidad locales}

Lo anterior pone de manifiesto que el diagnóstico «global-estructuralista» ha obviado la importancia y especificidad que presenta la comunidad local frente a otras escalas. Precisamente, ésta se encuentra en el hecho de constituir un «fenómeno social totalizador»(Köning, 1971). En ella se mantienen relaciones sociales más o menos estables que, a diferencia de las que tienen lugar en otras

(5) Diversos análisis han mostrado la importancia del capital cultural en la puesta en marcha de procesos de desarrollo, así como en procesos de cooperación y autorregulación de procesos colectivos (Keohane y Ostrom, 1995). 
escalas, constituyen la forma en que sigue desarrollándose la mayor parte de los vida cotidiana en las sociedades contemporáneas. Esto implica que las relaciones de vecindad de carácter local continúan siendo significativamente más importantes que las de carácter nacional o mundial, aún en un contexto caracterizado por la globalización y el aumento de la «acción a distancia» (Giddens, 1980).

Este hecho se manifiesta, al menos, de dos formas. Por un lado, la comunidad local es todavía uno de los objetos principales de orientación psicosocial, por el que se articula la identidad de los individuos. El hecho de que la vecindad local posibilite la interacción directa en mayor medida que la nacional o global, proporciona las bases para el mantenimiento de relaciones constantes e intensas y la creación de una identidad colectiva centrada en el territorio. Varios estudios empíricos muestran que laidentificación territorial de los individuos es principalmente local, siendo menor la nacional y casi inexistente la que transciende las fronteras estatales. En el caso español, por ejemplo, la mitad de los ciudadanos se identifican con el pueblo o ciudad en donde viven, una cuarta parte lo hace con España, menos de una quinta parte con su comunidad autónoma y menos del 10\% con su provincia o entidades supranacionales (6).

Este fenómeno ha hecho que algunos autores señalen la crisis de las identidades políticas tradicionales, tanto la territorial, esto es, la referida al estado nacional (Romanelli, 1991), como las que originaban la formación de partidos políticos, ligadas, como señaló Rokkan (1970), a valores de carácter universalista (la clase, izquierda-derecha, religioso-laico) (7). Se produce, en cambio, la potenciación de la que se articula localmente, sin que existan muestras de una mayor identificación con entidades de carácter supranacional (Diamanti, 1994: 409).

Por tanto, al igual que en los procesos de reestructuración económica, las subculturas de índole local toman cada vez más relevancia en la configuración de las identidades políticas en Occidente y en el desarrollo de procesos políticos,

(6) Sobre este asunto pueden consultarse los informes anuales del CIRES y ORIzO (1991: 167). Para el caso Italiano, SEgatTi muestra que en 1995 el 36\% de los italianos se identifican en primer lugar con su comunidad local, igual cantidad que lo hace con la nación (1995: 109-110). Para el caso europeo pueden hallarse datos en los estudios de opinión que se resumen en Stoetzel (1982: 55) y Ashford y Timms (1992).

(7) Entre otros, este aspecto ha sido destacado por Allum (1995: 79-121; 183-187), en donde pueden consultarse otras referencias. 
tanto en el ámbito local propiamente dicho, como en la estructuración de procesos políticos a mayor escala (8).

La importancia de la comunidad local como «sociedad total» también se muestra a través de la extensión o amplitud de las redes sociales interindividuales en la vida cotidiana. Para la mayoría de los individuos, estas siguen concentrándose en el ámbito local, o incluso en el vecinal. Las redes de socialidad y amistad, y su fuerte contenido de ayuda mutua, suelen articularse mediante factores de carácter territorial. Para los españoles, por ejemplo, estas redes se centran fundamentalmente en relaciones vecinales y familiares (9). A ello se une que el grado de movilidad del individuo medio sea bastante bajo, y que en todo caso se circunscriba a movimientos rotacionales que toman como punto de referencia el ámbito local (Bericat, 1994). Incluso en grandes ciudades, caracterizadas por el anonimato y un alto grado de heterogeneidad social, como en los casos de Milán o Madrid, la cercanía geográfica parece tener mayor peso que las características sociales e individuales en el establecimiento de relaciones sociales (Mutti, 1992; Ayuntamiento de Madrid, 1991).

Por otro lado, resulta evidente que las sociedades contemporáneas se caracterizan en su configuración ecológica por ser urbanas. La ciudad, como expresión tangible del fenómeno urbano, es su forma típica de comunidad de asentamiento (10). Han sido motor y lugar de los cambios sociales acontecidos

(8) En este sentido Köning apuntaba que «la mayoría se identifica con esa totalidad de la vida. En este aspecto la comunidad se convierte en la 'patria' en su sentido más estricto» (KöNING, 1971: 16). La existencia de sub-culturas de carácter territorial fue puesta de manifiesto por Trigilia en sus análisis sobre desarrollo local en el norte de Italia (Trigilia, 1980), así como por Bagnasco (1980). Este punto de vista a sido desarrollado posteriormente por el análisis político, como por ejemplo CACCIAGLI (1993) y LEPETIT Y OTROS (1993).

(9) Sobre este asunto pueden consultarse los datos que ofrece el CIRES en sus informes anuales.

(10) Según los datos del «Social Indicators of Development 1991-1992» del International Bank for Reconstruction and Development (The World Bank), la proporción de población urbana sobre el total ha ido aumentando de forma generalizada desde 1965 a 1990. Por ejemplo, en el caso español los datos para estos años eran 61'3\% y 78'4\% respectivamente. En Portugal, 23'9\% y 33'6\%, en Italia, 61'8\% y 68'9\%, en el Reino Unido, 81 '7\% y 89'1\%, en Egipto 40’7\% y 46’7\%, en Gabón, 21'2\% y 45'7\%, en Brasil, 50’4\% y $74{ }^{\prime} 9 \%$, o en Argentina, $76.1 \%$ y $86.3 \%$. 
en las sociedades modernas y pueden ser consideradas el locus del proceso civilizatorio, tanto en su dimensión positiva como negativa (11). Las ciudades han acogido y concentrado las principales modificaciones económicas, políticas y sociales que han tenido lugar en las sociedades de Occidente. Base del desarrollo comercial y la extensión de redes de intercambio a larga distancia durante la Edad Media y los inicios de la era moderna. Posteriormente, lo fueron del desarrollo y expansión industriales. Actualmente, cuando empieza a conformarse la «sociedad post-industrial», y la ciencia social diagnostica la crisis del modelo de desarrollo urbano-industrial, las ciudades siguen actuando como impulsoras del procesos civilizatorio mediante la concentración y vertebración de redes de comunicación y desarrollo tecnológico, adquiriendo la dimensión de «ciudad global» (Mazzelini, 1993).

Pero al mismo tiempo, las ciudades han mostrado la «otra cara» del proceso civilizatorio. En ellas se han concentrado, más que en ningún otro lugar, la degradación social y medioambiental. En ellas aparecen de una forma acentuada los problemas que enfrentan las sociedades contemporáneas (desigualdad y polarización sociales, desempleo, pobreza, marginación, delincuencia, contaminación acústica, escasez y degradación de la calidad de los recursos hídricos, polución, y un largo etcétera) (12). En suma, la ciudad ha acogido y desarrollado en su seno tanto las fuerzas impulsoras como las externalidades del proceso modernizador.

\section{Democratización: bienestar descentralizado y mixto}

El desarrollo del estado de bienestar, como intento de respuesta a algunos de esos problemas, ha hecho que los gobiernos locales tengan una posición relevante en el desarrollo de las políticas públicas. El aumento de la intervención estatal en la vida cotidiana de los ciudadanos mediante políticas de bienestar

(11) Una visión global del desarrollo de las ciudades y la influencia del fenómeno urbano en el desarrollo de los países occidentales puede consultarse en la obra de MumFord (1961).

(12) F. Engels, con su descripción sobre la situación de la clase obrera fue uno de los pioneros en mostrar la «otra cara» del proceso civilizatorio. Sobre los problemas de las ciudades europeas pueden consultarse Ayuntamiento de Madrid (1989) o Mingione (1994). Para el caso español en particular, cf. BERTOLO y OTROs (1988). 
social trastocó el sentido de la tradicional división del trabajo político entre gobierno central y gobiernos locales.

El esfuerzo de la acción estatal, sin menoscabo de su acción política tradicional o «high politics» (recaudación de impuestos, orden interno, defensa externa y relaciones diplomáticas), pasa a concentrase después de la Segunda Guerra Mundial en políticas públicas de carácter social o «low politics», las cuales venían siendo competencia, o cuando menos, eran desarrolladas por los gobiernos locales (Bulpitt, 1983). Perola extensión del estado de bienestar ha producido que la antigua «low politics» se haya convertido en la nueva «high politics» (Allum, 1995: 414-420). Con ello, los procesos políticos que tienen lugar en el ámbito local están cobrando una relevancia en el conjunto de la política nacional desconocida anteriormente. Indicador de ello pude ser que los ciudadanos dirijan en mayor medida sus demandas a los gobiernos municipales que a otros niveles superiores, con independencia de la distribución de competencias, así como la cada vez mayor relevancia que adoptan las elecciones municipales (13).

A la reflexión anterior puede añadirse que, tal y como indicaron los teóricos clásicos de la democracia, el ámbito local proporciona condiciones más favorables que escalas más extensas para el desarrollo de modelos de gobierno democráticos. Pero a diferencia de estos autores, que incidían en la constitución de politeyas democráticas autónomas, el debate actual acerca de la «democracia local» centra su atención en el papel que desempeñan los gobiernos locales tanto en los procesos de democratización que tienen lugar a escala nacional, como en las posibilidades de desarrollar procesos de participación ciudadana en los municipios.

En este sentido, se señala la imposibilidad de comprender y finalizar los procesos de democratización sin atender a las dinámicas que tienen lugar tanto a nivel mundial, como a nivel local (Archibugi y Held, 1995). En el análisis de la democratización de los países de la Europa oriental se incide, cada vez más, en

(13) Según el British Participatory Study el 44’2\% de las acciones políticas ejercidas por los ciudadanos británicos se orientan la gobierno local y sólo el 15'1\% al gobierno central (PARRY Y OTROS, 1992: 269). La importancia de las elecciones locales, y su papel en la estrategia de los partidos, ha sido puesta de manifiesto por GYFORD Y OTROS (1989) para el caso británico. Para el caso español puede consultarse CAPó (1991), BALME (1989) para Francia y BetTin (1993) para Italia. 
el hecho de que uno de los requisitos para la culminación de tales procesos a escala nacional es el establecimiento de formas de gobierno democráticas en el ámbito local (14). La constatación es similar por lo que respecta a la consolidación de las democracias africanas y centroamericanas (15).

En las democracias liberales de Europa y Norteamérica se insiste, tanto por parte del ámbito académico como del político y ciudadano, en la necesidad de establecer mecanismos que favorezcan la eficacia en la provisión de los servicios, así como en el desarrollo de modelos de gobierno que aumenten la participación de los ciudadanos en la toma de decisiones colectivas. En estas reflexiones el centro de atención viene siendo el ámbito local (16).

Con independencia del enfoque teórico o de las vías por las que se propone el desarrollo de modelos de democracia participativa, es lugar común señalar que el ámbito local es el lugar apropiado para su puesta en marcha (17). Las diversas propuestas señalan que este modelo de democracia consistiría en la complementación de los canales de participación electoral, propios de la democracia representativa y vía legítima de acceso al poder político, mediante mecanismos

(14) En el análisis de los procesos de democratización de los países de la Europa del Este se incide, cada vez en mayor medida, en este hecho, como por ejemplo señala STOKER (1991). En un estudio realizado por Miguel y Berland en 1991 se pone de manifiesto como las tres cuartas partes de los ciudadanos húngaros, polacos y checoslovacos creen que le gobierno local posee una gran influencia en los procesos de democratización que se desarrollan en sus países, incluso en mayor medida que el parlamento u otras instituciones políticas (Miguel y Berland, 1992: 86). Para una visión de conjunto se remite a BALDERSHEIM Y OTROS (1996).

(15) En el caso de Nigeria y Kenia cf. Olowu (1993) y NGaU (1993) respectivamente. Para una visión de los países caribeños KLAK y RULLi (1993).

(16) Diamanti señala que el interés académico por el ámbito local, lo que denomina «localismo», debe entenderse como la recepción de un consenso anteriormente existente entre los actores económicos y políticos acerca de las posibilidades de desarrollo económico y político que este ámbito muestra (Diamanti, 1994).

(17) En este sentido Wiliam A. Kelso señala que el «término democracia participativa es aplicado a diferentes variantes de teoría democrática, todas las cuales postulan que la devolución de poder a pequeñas unidades de gobierno traerá consigo la maximización de las oportunidades de participación pública» (1978: 175). Esta visón en ampliamente compartida por la teoría democrática contemporánea, véanse, por ejemplo, MACPERSHON (1991: 130), Pateman (1970: 19), Dahl (1992:384-385), Hirts (1994: 26), Barber(1986: 262) o HELD (1992: 313). 
que potencien la participación directa de la ciudadanía (18). Una fórmula mixta entre la forma contemporánea de democracia y la democracia clásica, sólo realizable en escalas reducidas, en las que la cercanía ente gobierno y ciudadanos permitiría el establecimiento de relaciones continuas e intensas.

De hecho, desde los años sesenta se vienen produciendo diversas experiencias que traducen en el plano político-institucional las ideas básicas del modelo de democracia participativa. Entre estas destacan las desarrolladas en municipios italianos y españoles en la forma de reglamentos de participación ciudadana, por los que se trata de regular, de forma estable, el acceso y participación de los ciudadanos o grupos de representación de intereses constituidos por éstos en los procesos de toma de decisiones que constituyen el flujo de la política municipal (Navarro, 1997, 1998).

Por último, existe un nuevo elemento en la discusión sobre el papel a desarrollar por los municipios en el proceso de democratización: las ventajas que presenta la descentralización de la gestión y provisión de servicios hacia los municipios frente a la centralización a nivel nacional. Se postula que en los primeros, al establecerse una relación más cercana y rutinaria con los ciudadanos que en otros niveles de gobierno, se da una mayor y más rápida adaptación de la acción pública al contexto social sobre el que ha de actuar. Además, frente a la descentralización a través de agencias gubernamentales, la que se realiza a través de los municipios ofrece la posibilidad de coordinar la provisión de servicios, de forma que la acción pública actúe integralmente sobre la multidi-

(18) Atendiendo a las vías concretas por las que diversos autores señalan pueda darse tal complementación, en términos generales cabe distinguir tres propuestas, a los que cabe denominar pluralista, deliberativa y expansionista. En la primera se trata de aumentar la interrelación entre los grupos y asociaciones y las instituciones representativas, de modo que los ciudadanos, a través de los primeros, ejerzan un mayor control entre períodos electorales (Kelso, 1978, DAHL, 1982, HIRTS, 1994). La estrategia deliberativa postula la creación de ámbitos en los que la ciudadanía pueda llegar a definir los intereses de la comunidad mediante la deliberación y la discusión públicas, como por ejemplo, la creación de «asambleas vecinales», de entre mil y cinco mil ciudadanos, con funciones deliberativas, e incluso legislativas (BARBER, 1986). La tercera estrategia ve en la expansión de la democracia desde el ámbito político a otras esferas sociales la base sobre la que desarrollar una democracia, aún más, una sociedad participativa, como medio por el que romper el círculo vicioso de la participación, en el que las desigualdades económicas y sociales se transfieren al ámbito político (PATEMAn, 1970; BobBio, 1984). 
mensionalidad de problemas que afectan a los ciudadanos (Clarke y Gaile, 1989).

Muestra de ello puede ser que, tal y como se ha indicado en el primer apartado, todos los gobiernos centrales de Unión Europea, a excepción del Reino Unido, tienden a descentralizar la provisión bienestar a través de los gobiernos municipales, en la forma de servicios sociales de carácter personal, así como lo referido al manteniemiento de condiciones mínimas de urbanización (Comisión Europea, 1988).

El hecho de que la descentralización acerque los procesos de toma de decisiones y la provisión de servicios a los ciudadanos ha planteado la posibilidad de ligar ambos objetivos, con el fin de aumentar la legitimidad y la eficacia de los sistemas democráticos contemporáneos. Y ello, tanto lo que se refiere a los gobiernos municipales en si mismos, como a la repercusión que ello puede tener sobre niveles de gobierno superiores, toda vez que los primeros, como locus de la provisión de servicios de bienestar, adquieren cada vez mayor relevancia política.

Pero la conjunción de democratización y descentralización está dando lugar a cierta reformulación en el desarrollo de las políticas públicas, y con ello, en el estado de bienestar. Este, tradicionalmente centrado en la acción pública directa sobre la ciudadanía, se abre para que agentes privados, con o sin ánimo de lucro, participen en la provisión de servicios de bienestar (Navarro, 1988). Esta gestión privada de intereses públicos, está conformando un «estado de bienestar mixto o pluralista», en el que participan tanto el estado, como empresas y entidades ciudadanas, para intentar procurar así una mayor flexibilidad y eficacia en la solución de los problemas que presentan las comunidades locales (19).

\section{Las aportaciones localistas al proceso de restructuración mundial}

Lo anterior permite concluir que existe un consenso generalizado acerca de la potencialidad del municipio como contexto adecuado para el desarrollo

(19) En Johnson (1990) se presentan los rasgos básicos del binestar mixto o pluralista, un sitema en el que estado, sector privado, asociaciones y redes de sociabilidad se complementan como proveedores de bienestar. BoRJA y CASTELl (1997: 107) han puesto de manifiesto la importancia del último sector, y en concreto el papel de las mujeres en la caso de las ciudades. 
económico, político y social en las postrimerias del siglo XX. Frente a la globalización, la comunidad local se sigue mostrando como el principal referente socio-espacial en la vida cotidiana. Desde un punto de vista económico surgen estrategias de desarrollo endógeno, centradas en las características e implicación de los actores locales. Estas intentan responder tanto a la nueva estructura de oportunidades resultante del proceso de globalización, como a las que se generan en el propio ámbito local. En la esfera política se vislumbra un proceso general de descentralización desde los gobiernos centrales a los gobiernos locales, así como intentos de desarrollar internamente en los municipios sistemas de gobierno que posibiliten tanto una provisión eficaz de servicios, como la participación de los ciudadanos en los procesos de decisiones colectivas que les afectan. En su conjunto, a esta tendencia que ofrece nuevas oportunidades para que los ciudadanos participen en el diseño de su futuro colectivo a partir del ámbito local, para la redefinición del papel de los gobiernos y sociedades municipales, es a lo que denominamos nuevo localismo.

En suma, puede señalarse que al mismo tiempo que las sociedades contemporáneas se ven atravesadas por procesos globales de carácter universal, albergan dinámicas locales que intentan solventar problemas generados tanto dentro como fuera de sus límites. Globalización y nuevo localismo pueden entenderse como dos procesos que, aún estableciendo tensiones entre si, articulan al mismo tiempo los procesos de reestructuración y desarrollo políticos, económicos y sociales que acontecen en las sociedades contemporáneas, generando nuevas oportunidades para la implicación de los ciudadanos en las decisiones que les afectan.

Ahora bien, para evitar los riesgos que puedan introducir ciertas lógicas localistas, y por tanto particularistas, en el sistema social y político, los beneficios de este proceso tensional deben canalizarse a través de reformas institucionales que encuentren nuevos puntos de equilibrio entre lo global y lo local. Lo que pasa, inevitablemente, por la integración de las lógicas locales en marcos de acción más amplios, así como en los procesos de toma de decisiones por los que se trata de regular políticamente el nuevo horizonte mundializado de nuestras sociedades. 


\section{Bibliografía}

Aglietta, M. (1974), Acumulación y crisis del capitalismo. México: FCE.

Allum, P. (1995), State and Society in Western Europe. London: Polity Press. Amin, S. (1972), Le développement inégal. Paris: Les Éditions de Minuit.

Ashford, S. y Timms, N. 1992. What Europe thinks. A study of Western European Values. Aldershot: Dartmouth.

Ayuntamiento De MadRID (1989), Desarrollo Europeo y estrategias de las grandes ciudades. Madrid: Ayto. de Madrid.

Ayuntamiento de MAdRID (1991), Encuesta sobre formas de vida de la población madrileña. Monografía II. La ocupación del tiempo libre. Las relaciones sociales y el asociacionismo. Madrid: Ayto. de Madrid.

Bagnasco, A. (1977), Tre Italie. La problematica territoriale dello sviluppo italiano. Bologna: il Mulino.

BAldersheim, H. y otros (1996): Local democracy and the processes of transformation in Esat-Central Europe, Oxford, Westview Press.

BaLme, R. (1989), «Councillrs, issue agendas y political action in tow French towns». Pp. 135-156 in Local Politics and Participation in Britain and France, Mabileau, A. y otros. Cambridge: Cambridge University Press.

Barber, J. (1984), Strong Democracy. Berkeley: Berkeley University Press.

Bericat, E. (1994), Sociología de la movilidad espacial. El senderismo nómada. Madrid: CIS.

Bertolo, C. y otros (1989), Crisis social de la ciudad. Madid: ALFOZ/ CIDUR.

BetTin (1993), Classe politica e città. Modena: CEDAM.

BLAIR, Ph. (1991), «Trends in local autonomy and democracy: reflections from an european perspective.» Local Government in Europe. Trends and development, Batley, R. y Stoker, G. (eds.). London: MacMillan.

Boвbio, N. (1984), Il futuro della democrazia. Torino: Einaudi.

BORJA, J. y CASTELL, M. (1997), Local y global. La gestión de las ciudades en la era de la información. Madrid: Taurus.

BulpitT, J. G. (1983), Territory and Power in the United Kingdom. Manchester: Manchester Univeristy Press.

CAPó, J. (1991), «Elecciones municipales, perono locales», en Revista Española de Investigaciones Sociológicas, 56, 143-164. 
Castells, M. (1976), The Urban Question. Cambridge: MIT Press.

Clarke, S. y. Gaile, G. (1989. «Moving towards entrepreneurial state and local economic development strategies: opportunities and barriers». Policy Studies Journal, 17, 574-598.

Clarke, S. (1993), «The New Localism: Local Politics in a Global Era». Pp. 121 in The New Localism. Comparative Urban Politics ina Global Era, Goetz, E. y Clarke, S. (eds.). London: Sage.

Cockburn, C. (1977), The Local State. London: Pluto Press.

Consejo DE Europa (1988), Allocation of power to the local and regional levels of government in the member states of the Council of Europe. Strasbourg: Council of Europe.

Diamanti, I. (1994), «Localismo». Ressegna Italiana di Sociologia, a. XXXV, 3, 401-424.

Dunleavy, P. (1980), Urban Political Analysis. London: MacMillan.

FrANK, A. (1972), Capitalismo y subdesarrollo en America Latina. Madrid: SigloXXI.

Giddens, A. (1990), The Consequences of Modernity, London: Polity Press. GyFORD, J. y OTROs (1989), The changing politics of local goverment. London: Unwim Hyman.

Hampton, W. (1991), Local Government and Urban Politics. London: Logman.

Hula, R. (1993), «The state reassessed: the privatization of local politics». Pp. 22-45 in The New Localism. Comparative Urban Politics in a Global Era, Goetz, E. y. Clarke, S. (eds.). London: Sage.

Johnson, N. (1990. El estado de bienestar en transición. La teoría y la práctica del pluralismo de bienestar. Madrid: Ministerio de Trabajo y Seguridad Social.

Kelso, W. A. (1978), American Democratic Theory. London: Greenwood Press.

Keohane, R. y Ostrom, E. (eds.) (1995), Local Commons and Global Interdependence. Heterogeneity and Cooperation in Two Domains. London: Sage.

KLAK, T. y RULLI, J. (1993), «Regimes of accumulation, the Caribbean Basin Initiative, and xport processing zones: scales of influence on carribbean development». The New Localism. Comparative Urban Politics in a 
Global Era, Goetz, E. y Clarke, S. (eds.). London: Sage.

KönING, R. (1971), Sociología de la comunidad local. Madrid: Fundación FOESSA.

LAFONTAINE, O. (1991), «Globalización de la economía y primacía de la política», en Alfoz, n. 90, 77-86.

LAMBI, C. y оTRos (1998), Copying with globalization, mimeo.

LIPIETZ, A. (1993), «Il locale e il globale: personalità regionale o interregionalità?». Pp. 41-60 in Globale/locale. Il contributo delle science sociali, Perulli, P. Milano: Franco Angeli.

Mazzoleni, Ch. (1993), «Città locale/cittá globale. Dello spazio dei luoghi allo spazio dei flussi e delle reti». Pp. 139-158 in Globale/locale. Il contributo delle science sociali, Perulli, P. (ed.). Milano: Franco Angeli.

Miguel, M. y Berland, M. (1992), «The future of democracy in Eastern Europe». Pp. 77-87 in Strategies of democratization, Vanhanen, T. (ed.). London: Crane Russak.

Mingione, E. (1994), «Polarización, fragmentación y marginalidad en las ciudades industriales». Pp. 97-122 in Clase, poder y ciudadanía, Alabart, A. y otros (eds.). Madrid: Siglo XXI.

Mumford, L. (1961), The city in the History. Orlando: Harcourt.

MutTI, A. (1992), Il buon vicino. Rapporti di vicinato nella metropoli. Bologna: il Mulino.

NAvarro YÁÑEz, C. J. (1997), Innovación democrática en el sur de Europa. Las experiencias municipales de Italia y España (1960-1995). Tesis doctoral inédita. Universidad Complutense de Madrid.

Navarro YÁÑEz, C. J. (1998), El nuevo localismo. Municipio y democraciua en la sociedad global, Córdoba, Servicio Publicaciones Diputación Provincial de Córdoba.

NGaU, P. (1993), «Political restructuring and the development process in Kenya». The New Localism. Comparative Urban Politics in a Global Era, Goetz, E. y Clarke, S. (eds.). London: Sage.

Olowu, D. (1993), «Local institutions and development: the nigerian experience». The New Localism. Compartive Urban Politics in a Global Era, Goetz, E. y Clarke, S. (eds.). London: Sage.

Orizo, F. A. (1991), Los nuevos valores de los españoles. Madrid: Editorial S.M. 
Parry, G.; Moyser, G. y Day, N. (1992), Political Participation and Democracy in Britain. Cambridge: Cambridge University Press.

Pérez SÁiz, J.P. (1997), «Entre lo global y lo local. Economías comunitarias en Centroamérica», en Sociología del Trabajo, n. 30, pp. 3-(19.

Piore, M. y. SABel, C.F. (1984), The second industrial divide. New York: Basic Books.

Pretecille, E. (1994), «Paradojas políticas de las restructuraciones urbanas, globalización de la economía y localización de lo político».Pp.61-96in Clase, poder y ciudadanía, Alabart, A. y otros (eds.). Madrid: Siglo XXI.

Rokkan, S. (1970), Citizens, Elections, Parties. Oslo: Universitetsforlaget.

RomanelLi, R. (1991), «Le radice istiriche del localismo italiano». Il Mulino, 4.

SASSEN, S. (1990), «Beyond city limits: a commentary». Pp. 237-242 in Beyond the city limits: urban policy and economic restructuring in compartive perspective, Logan, J. y Swanstrom, T. (eds.). Philadelphia: Temple University Press.

Segatti, P. (1995), «Una nazione di campaesani. Localismo e sentimento nazionale.» Pp. 105-138 in Sulla soglia del cambiamento, Parisi, A. y Schaedee, H. (eds.). Bologna: Il Mulino.

Stoetzel, J. (1983), ¿Qué pensamos los europeos?, Madrid: Editorial MAPHRE.

STOKER, G. (1991), «Introduction: Trends in European Local Governments». Local Government in Europe. Trends and developments, Batley, R. y Stoker, G. (eds.). London: MacMillan.

TrigiLIa (1981), Le subculture politiche territoriale. Milano: Feltrinelli. 